\title{
Flächendeckende Versorgung des akuten Schlaganfalls im Jahr 2016 und 2017 durch Neuro-Radiologen mittels mechanischer Thrombektomie in Deutschland anhand des DeGIR/DGNR-Registers
}

\author{
On the Basis of the DeGIR/DGNR Register Nationwide Care for Acute Ischemic Stroke \\ Patients in 2016 and 2017 Using Mechanical Thrombectomy by Radiologists and \\ Neuroradiologists
}

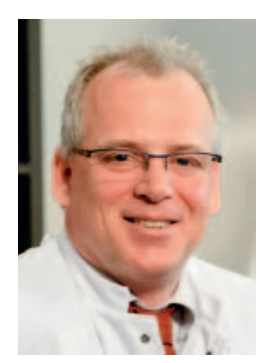

Ansgar Berlis

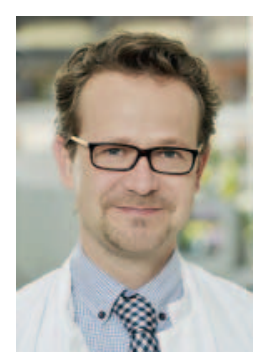

Dominik Morhard

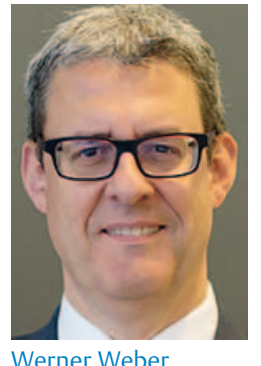

Werner Weber
Korrespondenzadresse

Prof. Ansgar Berlis

Diagnostische und Interventionelle Neuroradiologie,

Universitätsklinikum Augsburg, Stenglinstr. 2,

86156 Augsburg, Germany

Tel.: ++ 49/821/4002468

ansgar.berlis@klinikum-augsburg.de
Bibliografie

DOI https://doi.org/10.1055/a-0857-6694

Online-Publikation: 4.4.2019

Fortschr Röntgenstr 2019; 191: 613-617

(C) Georg Thieme Verlag KG, Stuttgart · New York

ISSN 1438-9029
Die akute Schlaganfallbehandlung hat im Jahr 2015 einen Paradigmenwechsel vollzogen. Die Standardtherapie der intravenösen rtPA-Behandlung (IVT) wurde auf Grundlage der neuen Studienlage ergänzt durch die mechanische Thrombektomie (MT), die bei Nachweis eines großen und relevanten Gefäßverschlusses der Hirngefäße der vorderen Zirkulation als Bridging-Therapie (MT und IVT) oder bei Kontraindikationen für die IVT als Monotherapie durchgeführt wird. In der Hermes-Analyse wurden 5 randomisierte Studien (MR CLEAN, ESCAPE, REVASCAT, SWIFT PRIME und EXTEND IA), die Daten zwischen 2010 und 2014 erhoben haben, in einer Metaanalyse ausgewertet [6]. Die Datenanalyse untermauert die sehr offen gehaltene S2k-Leitlinie zur Behandlung des akuten Schlaganfalls [1]. Hiernach profitieren Patienten bis zu 420 Minuten nach Schlaganfallbeginn von der MT, sodass das bislang starre 6-Stunden-Zeitfenster als starre Indikationsgrenze fällt. Die Studienlage beruht auf ca. 85 \% Behandlungen mit BridgingTherapie, sodass die Indikation für die IVT nach wie vor im Rahmen der Leitlinie besteht.

Der deutlich höhere Anteil an akuten Schlaganfallbehandlungen erfolgt mittels IVT als symptomorientierte Behandlung ohne Nachweis eines Gefäßverschlusses und ist damit meist eine unspezifische symptomorientierte Behandlung. Demgegenüber ist die MT eine spezifische verschlussorientierte Behandlung mit dem Ziel der gezielten Gefäßeröffnung [8]. Der klinische Erfolg der Methode korreliert mit dem Grad der Rekanalisation und der Zeit bis zur erfolgreichen Rekanalisation des Gefäßes.

Die flächendeckende Umsetzung dieser evidenzbasierten Methode hat in der stationären klinischen Versorgung dieser Patienten zu Veränderungen geführt. Das dichte Netzwerk von Stroke-Units (SU) nimmt jährlich zu. So hat sich die Zahl der SUs auf 309 erhöht und die Anzahl der überregionalen SU, die eine endovaskuläre 24/7 Schlaganfallversorgung mit MT vorhalten müssen, ist ebenfalls kontinuierlich auf 111 Einrichtungen gestiegen (Stand 1. März 2018) [4].

Eine zunehmende Anzahl radiologischer und neuroradiologischer Institute bieten die MT während der Regelarbeitszeit oder ganzjährig 24/7 an. Für die Zeit vor der Publikation der positiven Studienergebnisse zur MT haben Eyding et al. (2017) eine Auswertung der strukturierten Qualitätsberichte der Krankenhäuser der Jahre 2010 bis 2015 vorgenommen. Erfreulich sind der bundesweite Anstieg an rtPA-Behandlungen von $8 \%$ auf $12,2 \%$ und der Anstieg an MT von 0,7\% auf 3,1\% [5].

Bezüglich der Datenqualität dieser strukturierten Qualitätsberichte gibt es allerdings Einschränkungen. Die Veröffentlichung der dort registrierten Behandlungszahlen hinkt in der Regel zeitlich der tatsächlichen Entwicklung hinterher. Die Daten werden von den jeweiligen Kliniken freigegeben, ohne dass eine übergeordnete Überprüfung der Kodierung stattfindet. 
Das Problem der zuletzt für 2015 präsentierten DeGIR/DGNRDatenanalysen zur MT in Deutschland lag darin, dass die Datenbank für Modul E neben der MT auch Daten zur Schlaganfallbehandlung im Rahmen einer Vasospasmus-Behandlung sowie elektive Eingriffe wie Karotis-Stenting und die Behandlung intrakranieller Stenosen beinhaltet [3]. Ein weiteres Problem ist, dass die Dateneingabe freiwillig ist. Nicht alle Kliniken nehmen teil, Daten erscheinen nicht, weil unvollständig oder gar nicht eingegeben wurde. Damit spiegeln zwar die präsentierten Zahlen nicht die realen Behandlungszahlen wider, sie zeigen aber einen eindeutigen Trend.

Zum Teil bestehen erhebliche Differenzen zwischen realen und im Register aufgeführten Behandlungszahlen. Dies nehmen einige Teilnehmer des Registers zum Anlass, dass ihre Daten nicht öffentlich gemacht werden. Dieser Umstand ist zwar nachvollziehbar, zeigt aber auch die Sensibilität bezüglich einer nicht optimalen Klinikpräsentation im Vergleich mit umliegenden Kliniken, die ein vergleichbares Behandlungsspektrum anbieten. Dies wird vermutlich so lange bestehen bleiben, bis die Dateneingabe verpflichtend wird und eine sektorale Versorgung der Patienten umgesetzt wurde.

\section{Ist denn das Register repräsentativ und lohnt es sich, dies weiter zu unterstützen?}

Hier muss ein eindeutiges Ja stehen. Zum einen sind wir nach §137 SGB verpflichtet, Qualitätssicherung durchzuführen, und zum anderen werden zusätzliche Daten erfasst, die aktuell noch nicht Bestandteil dieser Publikation sind. Aktuell erhalten wir einen ausreichenden Überblick über die Versorgungslandschaft in Deutschland und wir erkennen an der nachfolgend aufgeführten Karte ( $\triangleright$ Abb. 1), dass wir dem Ziel der flächendeckenden Versorgung sehr nah sind. Ein Blick in die europäischen Nachbarländer zeigt, dass wir eine Flächendeckung aufweisen, die ihresgleichen sucht. In London wurde 2018 englandweit die erste Klinik mit 24/7-Versorgung in der Presse gefeiert.

\section{Wieso sollten wir alles unternehmen, um dieses Register fortzuführen und weiter zu verbessern?}

Das Register ist ein integrativer Bestandteil des Ausbildungskonzeptes für Interventionelle Radiologie und Neuroradiologie. Im Hinblick auf das Inkrafttreten des MDR (medical device regulation) im Mai 2020 sind Registerdaten eine wichtige Basis zur Beurteilung und Bewertung neuer Medizinprodukte. Registerdaten stellen die „real world“-Situation dar, wohingegen Studienergebnisse Daten auf dem Boden unterschiedlicher Selektionskriterien abbilden. Dies war ein Punkt, der auf dem AWMF-Treffen mit Beteiligung des BMG, G-BA, IQWiG, AWMF sowie Industrie und Fachgesellschaftsvertretern am 22.06.2018 in Berlin angesprochen wurde. In diesem Rahmen wurde darauf verwiesen, dass die Qualität eines Registers an der Beteiligung gemessen wird, die bei $95 \%$ eingegebener Behandlungen liegen sollte. Die daraus abzuleitende Konsequenz ist, dass zur Erreichung dieses Ziels die Unterstützung möglichst aller Kliniken erreicht werden sollte. Außerdem werden Dokumentationsassistenten benötigt, um eine korrekte und möglichst vollständige Dateneingabe zu gewährleisten. Wir sollten deshalb gemeinsam die Datenbank weiterentwickeln und anvisieren, dass wir in den nächsten Jahren eine stabile und möglichst vollständige Dokumentation, nicht nur zur akuten Schlaganfallbehandlung, erreichen, um für zukünftige Entwicklungen vorbereitet zu sein.

Die Registerzahlen aus Modul E für 2016 beziehen sich, entgegen der Zahlen von 2015, ausschließlich auf die Rekanalisation der Hirnarterien beim akuten Schlaganfall mittels mechanischer Thrombektomie. Die Spezifizierung der Dateneingabe wurde für 2017 weiter verbessert und für 2018 nicht zuletzt durch die neue Software komplett neu arrangiert. In $>$ Abb. 2 ist die Entwicklung der Dateneingabe für Modul E und MT für den Zeitraum 2014 2017 dargestellt. Waren es 2015 noch 169 Kliniken, die sich an den Eingaben zu Modul-E-Daten beteiligten, so konnte dies 2016 auf 178 und 2017 auf 204 Kliniken gesteigert werden. Die ModulE-Eingaben konnten im selben Zeitraum von 7302 (2015) auf 10793 (2016) und zuletzt auf 16759 (2017) in Deutschland gesteigert werden. Davon betreffen 8852 (2016) und 10680 (2017) akute ischämische Schlaganfallbehandlungen. Ein enormer Zuwachs, der bei einer geschätzten Fallzahl für Deutschland von ca. 13000 Patienten pro Jahr bedeutet, dass $68 \%$ bzw. $82 \%$ der potenziell zu behandelnden Patienten mit der DeGIR/DGNRDatenbank erfasst werden. Im Jahr 2016 haben von den 141 Kliniken, die der Veröffentlichung, die schriftlich von der DeGIRGeschäftsstelle eingeholt wurde, zugestimmt haben, insgesamt 125 Kliniken mindestens eine MT eingegeben. 107 Klinken haben zusätzlich der namentlichen Fallzahlpräsentation zugestimmt. Insgesamt beträgt die Gesamtfallzahl an MT der Kliniken, die einer Veröffentlichung zugestimmt haben, 8150 MT, während die verbleibenden 18 Kliniken im gleichen Zeitraum insgesamt 517 MT eingegeben haben. In $>$ Tab. 1 sind die Fallzahleingaben der Kliniken für 2016 und 2017 dargestellt. Jeweils $45 \%$ der Kliniken haben bis 50, und $55 \%$ mehr als 50 MTs eingegeben. Eyding et al. (2017) präsentierten ihre Daten aus 2015, bei denen $90 \%$ der Behandlungen an $44 \%$ der 209 Standorte durchgeführt wurden, während $10 \%$ der Behandlungen an $56 \%$ der Kliniken mit bedenklich geringer Fallzahl erfolgten. Die DeGIR/DGNR-Datenbank zeigt bereits für 2016, dass $96 \%$ der MTs > 30 Fälle an $75 \%$ der Kliniken erfolgt. Welche Schlussfolgerungen können wir daraus ziehen? Die Anzahl der Kliniken mit MT-Behandlungsmöglichkeit ist sehr hoch und eine flächendeckende Versorgung ist gewährleistet. Erfreulich ist der deutliche Anstieg an Kliniken mit 51 - 100 Behandlungen, der gewährleistet, dass an diesen Kliniken Interventionalisten ausgebildet werden können. Auffallend ist der relativ geringe Anteil an „High volume center“ mit über 250 Behandlungen, der zudem nicht unmittelbar mit der Bevölkerungsdichte korreliert. Gründe hierfür mögen Faktoren wie Ländergrenzen oder feststehende Kooperationen sein, sodass nicht unbedingt die nächstgelegene Klinik mit 24/7-neurointerventioneller Versorgung angefahren wird. Zukünftig wäre es wünschenswert, wenn verbindlich das nächstgelegene Zentrum mit Expertise angefahren wird.

Das Stroke-Unit-Netzwerk-Deutschland bietet eine nahezu flächendeckende Versorgung für den akuten Schlaganfall an. In einer Masterarbeit von Wünsch (2017) konnte gezeigt werden, 


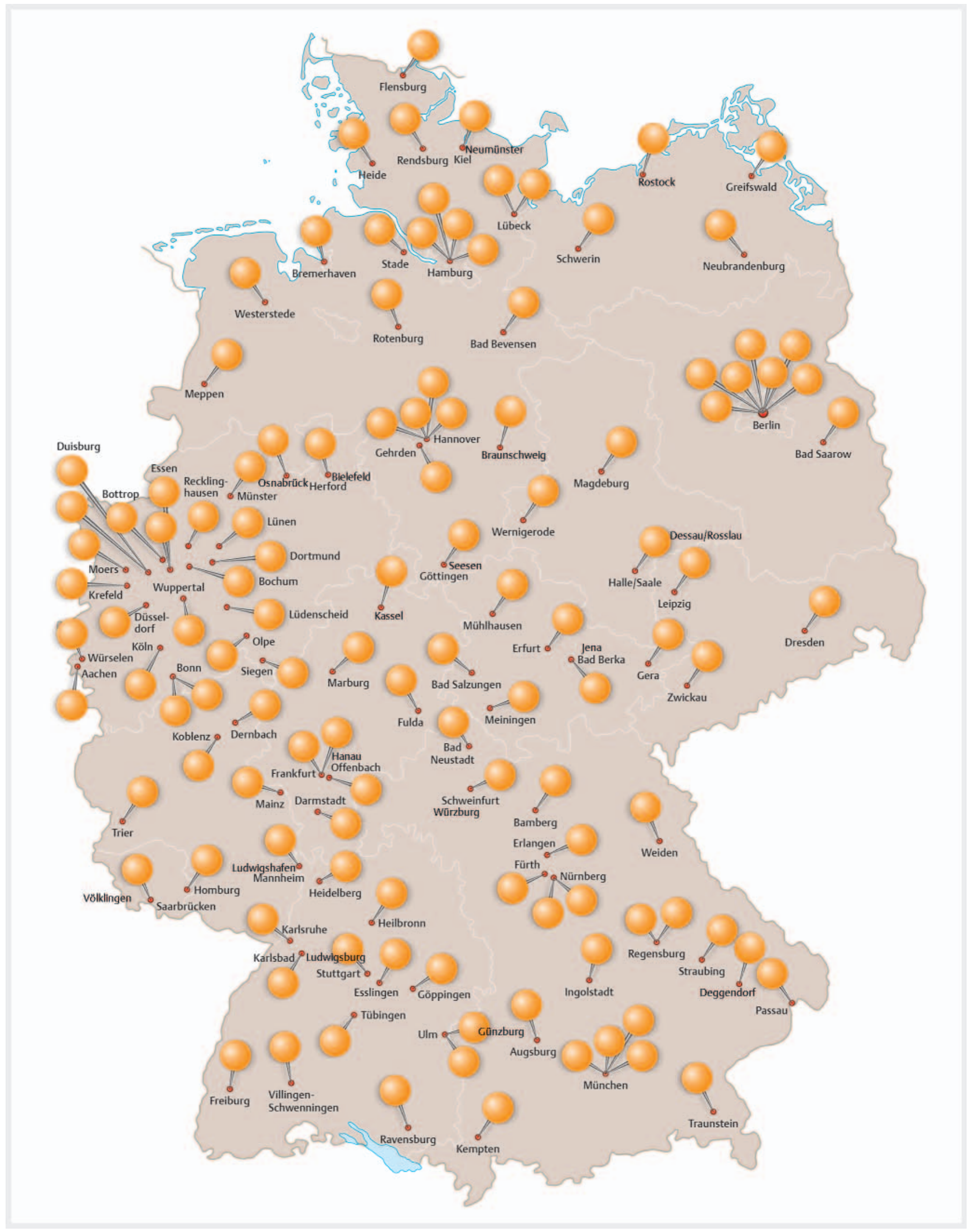

- Abb. 1 Bundesweite Verteilung von 125 Kliniken mit MT. Der Veröffentlichung haben für das Jahr 2016 insgesamt 141 von 178 Kliniken schriftlich zugestimmt, die freiwillig Modul-E-Daten in das DeGIR/DGNR-Register eingegeben haben. 
- Tab. 1 Clusteranalyse der teilnehmenden Kliniken nach Anzahl der eingegeben mechanischen Thrombektomien (MT) für die Jahre 2016 und 2017. Es zeichnet sich ein deutlicher Anstieg der Kliniken mit 51 - 100 Eingriffen pro Jahr ab.

\begin{tabular}{|l|l|l|l|l|l|}
\hline MT-Anzahl & $1-20$ & $21-50$ & $51-100$ & $101-150$ & $151-250$ \\
\hline $\begin{array}{l}\text { Kliniken 2016, die der Publikation von } \\
\text { Fallzahlen und Namen zugestimmt haben }\end{array}$ & 17 & 31 & 26 & 19 & 14 \\
\hline Kliniken 2017 & 35 & 35 & 49 & 19 & 13 \\
\hline
\end{tabular}

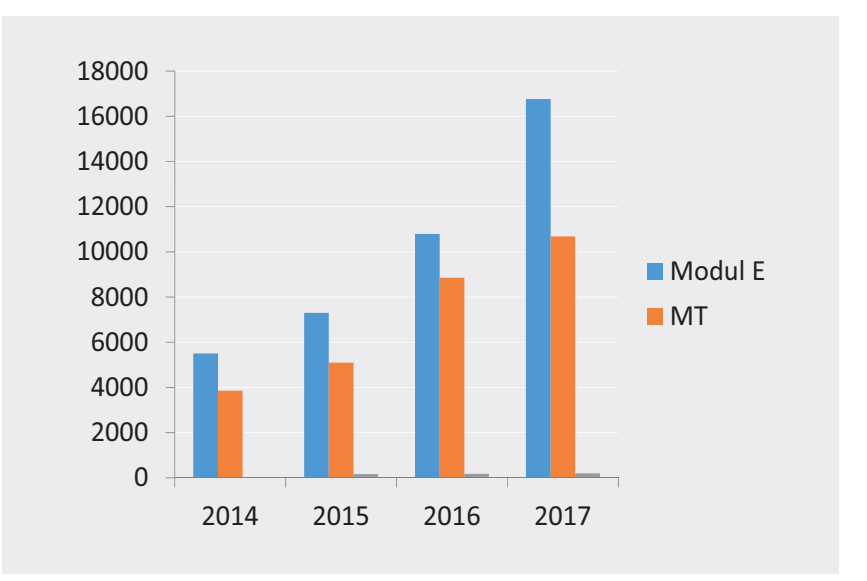

- Abb. 2 Entwicklung der Dateneingaben für Modul E und MT im Zeitraum von 2014 bis 2017. Während die MT-Daten für 2014 und 2015 intraarterielle Rekanalisationen inklusive intraarterieller Lysen beinhalten, beziehen sich die Daten für 2016 und 2017 ausschließlich auf die MT.

dass bei einer bodengebundenen Transportzeit von 45 Minuten in eine überregionale Stroke-Unit mit einem 24/7-Angebot eine $85 \%$ ige und bei 60 Minuten Transportzeit eine 95,5\%ige Abdeckung der deutschen Bevölkerung erreicht wird [9]. Bei der bodengebundenen Transportzeit von 45 Minuten in das nächstgelegene Zentrum könnten lediglich 240 Patienten (1,5\% aller Patienten) deutschlandweit nicht zeitgerecht behandelt werden. Dies untermauert die Forderung, dass Patienten in das nächstgelegene Zentrum verbracht werden.

Um möglichst vielen Patienten die akute Schlaganfallbehandlung mittels MT zukommen zu lassen, ist die Gewährleistung einer flächendeckenden Versorgung seit der Veröffentlichung der bekannten Studien zunehmend in der Diskussion. Begehrlichkeiten sind nicht nur von Kardiologen, sondern auch von anderen Fächern zu vernehmen. Eine Behandlung, die in Studien durch erfahrene Radiologen und Neuroradiologen durchgeführt wurde, mit geringer Komplikationsrate und hoher Erfolgsrate, kann nicht einfach auf andere Berufsgruppen ohne interventionelle Fertigkeiten an den Hirngefäßen und diagnostische Kenntnisse beim Schlaganfall transferiert werden. Die Methode ist auch erfolgreich, weil sie von Interventionalisten durchgeführt und gelehrt wird, die jahrelange Erfahrung in der Behandlung neurovaskulärer Erkrankungen haben. Hohe Fallzahlen sind erforderlich, um diese Expertise zu erhalten und auszubauen. Weiterhin muss an das Komplikationsmanagement gedacht werden. Bei immer älter werdenden Patienten mit komplexen Sondierungen der elongier- ten und verkalkten Zugangsgefäße stellen sich zum Teil erhebliche Anforderungen an die Durchführung der MT.

\section{Auswirkungen der aktuellen Studienlage auf die MT-Versorgung}

Die DAWN-Studie konnte zeigen, dass eine Schlaganfallbehandlung mit MT und i.v. rtPA in ausgesuchten Fällen bis 24 Stunden nach Symptombeginn der i.v. rtPA-Behandlung allein überlegen ist (mRs $0-2: 48 \%$ vs. $13 \%$ ) [7]. Ein guter Verlauf ist zwar eher in der Zeit von 6-12 Stunden (54 vs. 20\%) als nach $12-24$ Stunden (44 vs. $8 \%$ ) zu erwarten, der absolute Vorteil gegenüber der konservativen Behandlung steigt aber sogar in der Subgruppe (mit dem Zeitfenster 12 - 24 Stunden) an. Die DAWN-Studienergebnisse werden durch die zuletzt publizierten Ergebnisse der DEFUSE3-Studie bestätigt [2]. Die Behandlung erfolgte bis ins 16-Stunden-Zeitfenster und wurde aufgrund besserer Ergebnisse der MT nach Einschluss von 182 von geplanten 476 Patienten im September 2017 abgebrochen. Die Konsequenz wird sein, dass wir zukünftig mehr Patienten interventionell behandeln werden, sofern sie die bildmorphologischen Kriterien erfüllen, welche eine Behandlung auch nach 6 Stunden rechtfertigen.

Die Hermes-Analyse hat außerdem zeigen können, dass die MT auch ohne i.v. rtPA-Behandlung sehr gute und vergleichbare Ergebnisse zur Bridging-Therapie erreicht [6]. Dies heißt im Umkehrschluss nicht, dass wir zukünftig auf die i. v. rtPA verzichten, sondern dass bei jeder Kontraindikation für i. v. rtPA die MT als alleinige Therapie durchgeführt werden soll. Der Vergleich MT allein versus i. v. rtPA plus MT, mit dem sich aktuell randomisierte Studien beschäftigen, wird zu dieser Thematik möglicherweise weiteren Aufschluss geben. Wir sind auf die Ergebnisse gespannt.

Weitere Subgruppenanalysen o. g. HERMES-Analyse betreffen die Behandlung von peripheren Verschlüssen der intrakraniellen Gefäße, z. B. im M2-Segment. Die Ergebnisse erreichen aufgrund der mäßigen Fallzahl kein Signifikanzniveau, zeigen aber, dass die Komplikationen nicht erhöht sind und ein Trend zu guten Ergebnissen, vergleichbar den proximalen Verschlüssen, besteht. Betrachtet man die interventionelle Praxis zu diesem Thema, dann lässt sich der Trend beobachten, wonach immer häufiger und erfolgreich die MT auch bei Verschluss eines strategischen Gefäßastes praktiziert wird. Aus funktioneller Sicht liegt hier kein Unterschied zu einem M1-Verschluss vor.

Die weitere Entwicklung wird zeigen, dass wir durch die dargestellten Zusammenhänge in Zukunft mehr Patienten sinnvoll mit MT allein oder in Kombination mit i. v. rtPA versorgen können. Hierzu ist eine strukturierte Ausbildung der Neuro-Interventiona- 
listen genauso wichtig wie eine funktionierende Struktur innerhalb zertifizierter SUs. Die Patienten profitieren von professionellen Behandlungsteams, die routiniert und abgeklärt die Abläufe von der stationären Aufnahme bis zur Frührehabilitation begleiten und durchführen.

Literatur

[1] Akuttherapie des ischämischen Schlaganfalls, Ergänzung 2015 - Rekanalisierende Therapie. S2k-Leitlinie der Deutschen Gesellschaft für Neuroradiologie e.V. http://www.awmf.org/leitlinien/detail/I/030-140.html (Abruf vom 13.03.2018)

[2] Albers GW, Marks MP, Kemp S et al. Thrombectomy for Stroke at 6 to 16 Hours with Selection by Perfusion Imaging. N Engl J Med 2018; 378 : 708 718

[3] Berlis A, Weber W. Flächendeckende Akutversorgung von Schlaganfallpatienten durch die (Neuro-) Radiologie ist gewährleistet. Fortschr Röntgenstr 2017; 189: 303-308
[4] Deutsche Schlaganfallgesellschaft (DSG). Stroke Units. http://www.dsginfo.de/stroke-units/stroke-units-uebersicht.html (Abruf vom 13.03.2018)

[5] Eyding J, Bartig D, Kitzrow M et al. Gestaltung und Organisation der akuten endovaskulären Schlaganfalltherapie - Implikationen aus dem Neurovaskulären Netz Ruhr. Akt Neurol 2017; 44: 458-465

[6] Goyal M, Menon BK, van Zwam WH et al. Endovascular thrombectomy after large-vessel ischaemic stroke: a meta-analysis of individual patient data from five randomised trials. Lancet 2016; 387: 1723-1731

[7] Nogueira RG, Jadhav AP, Haussen DC et al. Thrombectomy 6 to 24 Hours after Stroke with a Mismatch between Deficit and Infarct. N Engl J Med 2018; 378: $11-21$

[8] Von Kummer R. Trials on ischemic stroke treatment: mission accomplished? Neuroradiology 2018; 60: 127-128

[9] Wünsch A. Flächendeckende interventionelle Versorgung von Schlaganfällen. Masterarbeit. Universität Augsburg. 2017

ERRATUM 20.08.2019

Berlis A, Morhard D, Weber W. Flächendeckende Versorgung des akuten Schlaganfalls im Jahr 2016 und 2017 durch Neuro-Radiologen mittels mechanischer Thrombektomie in Deutschland anhand des DeGIR/DGNR-Registers. Fortschr Röntgenstr 2019; doi:10.1055/a-0857-6694, Seite 613-617

Im Editorial „Ansgar Berlis, Dominik Morhard, Werner Weber, Flächendeckende Versorgung des akuten Schlaganfalls im Jahr 2016 und 2017 durch Neuro-Radiologen mittels mechanischer Thrombektomie in Deutschland anhand des DeGIR/DGNR-Registers“ wurden in Abbildung 1 „Deutschlandkarte“ nicht alle (neuro-)radiologischen Zentren, die im Erhebungszeitraum 2016-2017 mechanische Thrombektomien erbracht haben, erfasst. Die Karte ist auf thieme-connect.de aktualisiert. Der Verlag bittet das Versehen zu entschuldigen. 